\title{
Structure and evolution of the Fusarium mating type locus: New insights from the Gibberella fujikuroi complex
}

\author{
Simon H. Martin ${ }^{1}$, Brenda D. Wingfield ${ }^{1}$, Michael J. Wingfield ${ }^{2}$ and Emma T. Steenkamp ${ }^{2}$
}

(1) Department of Genetics, Forestry and Agricultural Biotechnology Institute, University of Pretoria, Pretoria 0002, South Africa.

(2) Department of Microbiology and Plant Pathology, Forestry and Agricultural Biotechnology Institute, University of Pretoria, Pretoria 0002, South Africa.

\section{Brenda D. Wingfield}

Email: brenda.wingfield@fabi.up.ac.za

\begin{abstract}
Mating type genes are central to sexual reproduction and compatibility in Ascomycete fungi. However the "MAT" loci experience unique evolutionary pressures that can result in rapid divergence and enhanced inter-specific gene-flow (lateral gene transfer). In this study, molecular evolution of MAT loci was considered using the genus Fusarium (Teleomorph: Gibberella) as a model. Both MAT1-1 and MAT1-2 "idiomorphs" from eleven species of the Gibberella fujikuroi species complex were sequenced. Molecular evolution of the MAT loci from these heterothallic (self-sterile) species was compared with that of the $M A T$ loci from nine homothallic (self-fertile) species in the Fusarium graminearum species complex. Although Fusarium has previously been thought to have the same complement of four MAT genes that are found in Neurospora, we found evidence of a novel gene, MAT1-2-3, that may be specific to the Hypocreales. All MAT genes share a similar set of cis-regulatory motifs, although homothallic species might have recruited novel regulatory elements, which could potentially facilitate alternate expression of MAT1-1-1 and MAT12-1. Fusarium MAT loci displayed evidence consistent with historical lateral gene-flow. Most notably, the MAT1-1 idiomorph of Fusarium sacchari appears to be unrelated to those of other species in the G. fujikuroi complex. In general, Fusarium MAT genes are highly divergent. Both positive selection and relaxed selective constraint could account for this phenomenon. However, the extent of both recombination and inter-specific gene-flow in the MAT locus also appears to affect the rate of divergence.
\end{abstract}

\section{Introduction}

In heterothallic (self-sterile) Ascomycetes, sexual reproduction can occur only between individuals of opposite mating type. The two mating types are determined by dissimilar "idiomorphic" forms of the mating type (MAT) locus. The dissimilarity between the two idiomorphs is maintained because this specific portion of the genome does not pair up and undergo homologous recombination during meiosis (Coppin et al., 1997; Kronstad and Staben, 1997). By convention, the idiomorphs are referred to as MAT1-1 and MAT1-2 (Turgeon and Yoder, 2000).

The genes encoded by the MAT locus are putative transcription factors thought to control sexual development and to regulate the expression of downstream, mating-type-specific genes (Coppin et al., 1997; Shiu and Glass, 2000). The gene composition of the locus vary can dramatically among species, but there are two MAT genes that are consistently found in filamentous Ascomycetes. 
MAT1-1 always contains a gene called MAT1-1-1, which encodes a protein homologous to MATa1 of Saccharomyces cerevisiae. This protein has a unique motif called the a-box. MAT1-2 always contains a gene called MAT1-2-1 that encodes a protein with a high-mobility-group (HMG) DNAbinding domain.

In homothallic (self-fertile) Ascomycetes sexual reproduction can occur between any two individuals. These species carry homologues of both MAT1-1-1 and MAT1-2-1 genes in the same genome (with the possible exception of a few Neurospora species (Glass et al., 1988; Glass and Smith, 1994). Coppin et al. (1997) proposed that self-fertility may be achieved via alternate expression of genes for one or the other mating type in distinct cells. Homothallic species would thus require a mechanism for independent regulation of MAT1-1-1 and MAT1-2-1 (Coppin et al., 1997). In certain Ascomycete genera, it is clear that homothallic species have arisen from heterothallic ancestors (e.g. Yun et al., 1999; O'Donnell et al., 2004). However, in many other genera, it is likely that multiple independent transitions from heterothallism to homothallism and vice-versa have occurred (reviewed by Lee et al., 2010).

Mating type genes have been found to be highly divergent between species (Cisar et al., 1994; Arie et al., 1997; Turgeon, 1998; Wik et al., 2008), yet they may be strongly conserved within species (Turgeon, 1998). Rapid diversification of sex-related proteins has been observed in many plants and animals, and the forces of sexual selection, sexual conflict and reinforcement have all been proposed as possible causes (reviewed by Civetta and Singh, 1998; Swanson and Vacquier, 2002; Clark et al., 2006). The ability to reproduce asexually adds a layer of complexity to this situation. Selection against the accumulation of detrimental mutations in MAT loci could at times be lacking. In Neurospora, Wik et al. (2008) found the MAT genes of heterothallic species to be under positive (diversifying) selection. However, the $M A T$ genes of homothallic species appeared to have diverged under a lack of selective constraint, and some contained premature stop codons. It was proposed that MAT genes may, therefore, be dispensable in homothallic Neurospora spp.

An important possibility that may have been overlooked is that the distinct genetic organization of the MAT loci could also shape their evolution. In heterothallic species, the effective population size of each idiomorph is half that of the rest of the genome (assuming equal mating type frequencies). This could cause reduced within species diversity, but enhanced inter-specific diversity as a result of genetic drift (Charlesworth, 2009). Unlike heterothallic MAT idiomorphs, homothallic MAT loci can presumably recombine freely during meiosis. The extent of recombination experienced by a locus could affect the efficiency of selection (Birky and Walsh, 1988) as well as the actual nucleotide substitution rate (Vicoso and Charlesworth, 2009). It is, therefore, important to distinguish between the effects of adaptive and non-adaptive forces on the patterns of diversity in MAT loci.

Recent research has shown that phylogenies constructed using Neurospora MAT genes are in conflict with species trees (Strandberg et al., 2010). It was proposed that reproductive genes may be more predisposed to inter-specific gene-flow than others. Transfer of sexual genes through hybridization could restore sexuality to populations in which it has been lost, as was observed in Ophiostoma (Paoletti et al., 2006). It has not been established whether homothallic species display similar characteristics (Strandberg et al., 2010). It could in fact be more difficult to detect lateral transfer in homothallic than heterothallic MAT loci. In the latter group, the lack of recombination ensures that the phylogenetic signal of hybridization or lateral transfer is not diluted by exchange of genetic material between "native" and introgressed alleles (Chaturvedi et al., 2002; Devier et al., 2010).

A useful model in which to study MAT locus evolution is found in the genus Fusarium (Teleomorph: Gibberella). Heterothallic Fusarium spp. such as those in the well-known Gibberella fujikuroi species complex, have three genes in the MAT1-1 idiomorph (MAT1-1-1, MAT1-1-2 and 
MAT1-1-3) and a single gene in the MAT1-2 idiomorph (MAT1-2-1) (Arie et al., 1999; Yun et al., 2000). These four MAT genes are homologous to the four MAT genes of the model species Neurospora crassa (Yun et al., 2000), where they are found in the same arrangement (Glass et al., 1990; Staben and Yanofsky, 1990). In the homothallic Fusarium graminearum complex, in which homothallism has a monophyletic origin (O’Donnell et al., 2004) all four MAT genes are adjacent within the MAT locus (Yun et al., 2000). Homothallism is thought to have arisen through an unequal crossover between MAT idiomorphs in a heterothallic ancestor (O'Donnell et al., 2004). Unlike Neurospora, there is no evidence that MAT genes are dispensable in the homothallic Fusarium spp. Knock-out strains of F. graminearum lacking either the MAT1-1 or MAT1-2 genes can only outcross in a heterothallic manner (Lee et al., 2003). A previous study of $M A T$ locus evolution in the $F$. graminearum complex (O'Donnell et al., 2004) yielded results in conflict with the Neurospora model: reduced divergence in $M A T$ genes and few phylogenetic inconsistencies. However, there has been no thorough, equivalent investigation of $M A T$ locus evolution in a monophyletic, heterothallic, Fusarium lineage. Interestingly, an earlier phylogeny constructed using short MAT sequences from species in the heterothallic G. fujikuroi complex did yield an unexpected topology for MAT1-1 (Steenkamp et al., 2000), but this was not investigated further.

In this study MAT locus structure and evolution in the G. fujikuroi complex has been studied in depth, allowing a thorough comparison between heterothallic and homothallic Fusarium species. The aims were to (1) identify and compare evolutionarily conserved regions and cis-regulatory motifs in the MAT loci of heterothallic and homothallic Fusarium spp.; (2) investigate whether phylogenetic conflicts exist between the MAT and species trees; and (3) compare patterns of molecular evolution in both coding and non-coding portions of heterothallic and homothallic loci. To achieve these aims, the complete MAT1-1 and MAT1-2 idiomorphs of eleven species in the $G$. fujikuroi complex, including nine mating populations (A-I), were sequenced.

\section{Materials and methods}

\section{Fungal isolates}

Twenty-two Fusarium isolates, a MAT1-1 and a MAT1-2 isolate for each of eleven species (Table 1), were used in this study. For the nine mating populations, the isolates used represent the standard MAT1-1 and MAT1-2 mating type tester strains. For two additional species, F. subglutinans group 1 and F. mangiferae, isolates characterized by Steenkamp et al. (2002) and Britz et al. (2002), respectively, were used. DNA was extracted from all isolates using the procedure described by Steenkamp et al. (1999).

\section{DNA sequencing}

Using published MAT1-1 and MAT1-2 idiomorphs of F. oxysporum and F. verticillioides (Yun et al., 2000) and their genome sequences (GenBank accession numbers: AAXH01000548 and AAIM02000073, respectively), PCR primers were designed in conserved regions to amplify overlapping fragments covering the length of both idiomorphs (Fig. 1 and Table A1). Reaction mixtures were $25 \mu \mathrm{l}$ in volume and contained $4 \mathrm{ng} / \mu 1$ template DNA, $1.5 \mathrm{mM} \mathrm{MgCl}, 0.4 \mu \mathrm{M}$ of each primer, $1 \mathrm{mM}$ deoxynucleotide triphosphates $(0.25 \mathrm{mM}$ of each), and $0.05 \mathrm{u}$ (units)/ $\mu 1$ SuperTherm DNA Polymerase and reaction buffer (Southern Cross biotechnology [Pty.] Ltd., Cape Town, South Africa). The PCR cycling conditions consisted of an initial denaturation at $94{ }^{\circ} \mathrm{C}$ for $60 \mathrm{~s}$; thirty cycles of denaturation at $94{ }^{\circ} \mathrm{C}$ for $30 \mathrm{~s}$, annealing for $30 \mathrm{~s}$ and extension at $70{ }^{\circ} \mathrm{C}$; followed by a final extension step at $70{ }^{\circ} \mathrm{C}$ for $10 \mathrm{~min}$. All PCR amplifications from genomic DNA were performed using the same protocol, except for annealing temperatures that depended on the primers used (Table A1) and extension times that depended on the expected length of the amplicons. Extension times were calculated by adding 1 min for each $\mathrm{kb}$ of sequence amplified. 
Table 1. Fusarium isolates in the Gibberella fujikuroi species complex used in this study.

\begin{tabular}{|c|c|c|c|c|}
\hline \multirow[t]{2}{*}{ Fusarium spp. } & \multicolumn{4}{|l|}{ Isolate ${ }^{\mathrm{a}}$} \\
\hline & $\begin{array}{l}\text { Mating } \\
\text { population }\end{array}$ & Mating type & MRC no. & KSU no. ${ }^{c}$ \\
\hline \multirow[t]{2}{*}{ F. verticillioides } & \multirow[t]{2}{*}{ A } & MAT-1 & 8559 & A-00149 \\
\hline & & MAT-2 & 8560 & A-00999 \\
\hline \multirow[t]{2}{*}{ F. sacchari } & \multirow[t]{2}{*}{ B } & MAT-1 & 8552 & B-03853 \\
\hline & & MAT-2 & 8551 & B-03852 \\
\hline \multirow[t]{2}{*}{ F. fujikuroi } & \multirow[t]{2}{*}{ C } & MAT-1 & 8532 & C-01993 \\
\hline & & MAT-2 & 8534 & C-01995 \\
\hline \multirow[t]{2}{*}{ F. proliferatum } & \multirow[t]{2}{*}{ D } & MAT-1 & 8549 & D-04854 \\
\hline & & MAT-2 & 8550 & D-04853 \\
\hline \multirow{2}{*}{ F. subglutinans (group 2) } & \multirow[t]{4}{*}{$\mathrm{E}$} & MAT-1 & $8553 / 6483$ & E-00990 \\
\hline & & MAT-2 & $8554 / 6512$ & E-02192 \\
\hline \multirow[t]{2}{*}{ F. subglutinans (group 1) } & & MAT-1 & 1084 & \\
\hline & & MAT-2 & 7828 & \\
\hline \multirow[t]{2}{*}{ F. thapsinum } & \multirow[t]{2}{*}{$\mathrm{F}$} & MAT-1 & 8558 & F-04094 \\
\hline & & MAT-2 & 8557 & F-04093 \\
\hline \multirow[t]{2}{*}{ F. nygamai } & \multirow[t]{2}{*}{ G } & MAT-1 & 8546 & G-05111 \\
\hline & & MAT-2 & 8547 & G-05112 \\
\hline \multirow[t]{2}{*}{ F. circinatum } & \multirow[t]{2}{*}{$\mathrm{H}$} & MAT-1 & 7488 & H-10847 \\
\hline & & MAT-2 & 6213 & $\mathrm{H}-10850$ \\
\hline \multirow[t]{2}{*}{ F. konzum } & \multirow[t]{2}{*}{ I } & MAT-1 & 8545 & I-11616 \\
\hline & & MAT-2 & 8544 & I-11615 \\
\hline \multirow[t]{2}{*}{ F. mangiferae } & \multirow[t]{2}{*}{$\mathrm{N} / \mathrm{A}$} & MAT-1 & $8092 / 8093$ & X4382 \\
\hline & & MAT-2 & 7559 & 11,781 \\
\hline
\end{tabular}

a Mating populations are represented by the standard MAT-1 and MAT-2 "tester" strains (Kuhlman, 1982; Leslie, 1991, 1995; Klittich and Leslie, 1992; Klaasen and Nelson, 1996; Klittich et al., 1997; Britz et al., 1999; Britz et al., 2002; Zeller et al., 2003; Leslie et al., 2005).

b Medical Research Council (MRC), Tygerberg, South Africa.

$c$ Department of Plant Pathology. Kansas State University (KSU), Manhattan, Kansas, USA.

The complete idiomorphs were amplified using primers MFC-R1 and MFC-L1, located in the 5' and 3' flanking regions respectively. In cases, where this PCR failed, MFC-L1 was substituted with a primer just inside the 30 end of the idiomorph (M1C-2 for MAT1-1 and M2C-4 For MAT1-2). Products were analyzed by electrophoresis in a $1 \%$ agarose gel at $20 \mathrm{~V} / \mathrm{cm}$ to verify purity and fragment size. MAT1-1 and MAT1-2 products were pooled to be sequenced together. Pools consisted of products from distinct species to avoid confusion in assembly arising from homology in the flanks. 454 sequencing was performed by Inqaba Biotech (Pretoria, South Africa). The pools were prepared separately using adapters carrying distinct identifiers, and sequenced together in one lane on a Roche GS-FLX Platinum sequencer. 


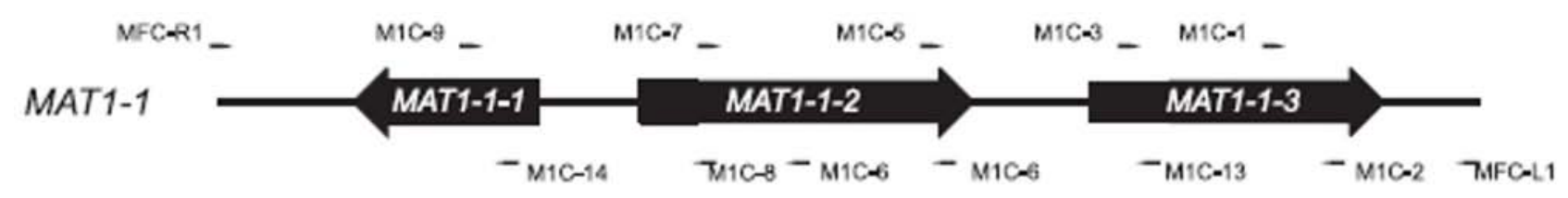

MAT1-2

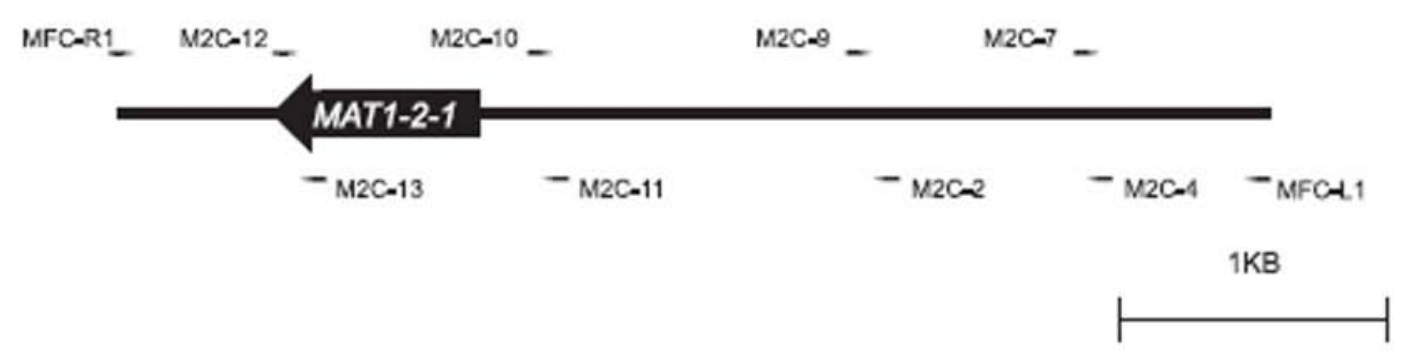

Figure 1. Binding sites of primers used to amplify MAT1-1 and MAT1-2 from G. fujikuroi species. Small arrows represent primer binding sites. Large block arrows represent MAT genes.

To fill in gaps as well as verify areas of low pyro-sequence coverage, PCR products were amplified using the appropriate primer pairs (Fig. 1). For F. proliferatum in which all PCRs incorporating the 30 MFC-L1 primer were unsuccessful, an additional primer, MFC-R2, was designed based on the 30 flank region of close relatives $F$. fujikuroi and F. mangiferae as a substitute for MFC-L1. Products were cloned in Escherichia coli using the pGEM $^{\circledR}$-T Easy Vector cloning system (Promega Corporation, Madison, WI). Cloned inserts were then amplified directly from colonies using the primers SP-6 (5'-ATTTAGGTGACACTATAG-3') and T-7 (5'-TAATACGACTCACTATAGGG-3'). This PCR reaction mixture was $25 \mu 1$ and contained $0.051 / \mu 1$ FastStart Taq DNA Polymerase (Roche Diagnostics, Mannheim, Germany), $1 \times$ Faststart buffer with $\mathrm{MgCl}_{2}, 1 \mathrm{mM}$ deoxynucleotide triphosphates $(0.25 \mathrm{mM}$ of each), $0.4 \mu \mathrm{M}$ of each primer and one bacterial colony. The colony PCR reaction conditions were as follows: Denaturation at $94{ }^{\circ} \mathrm{C}$ for $5 \mathrm{~min}$; followed by thirty cycles of denaturation at $94{ }^{\circ} \mathrm{C}$ for $30 \mathrm{~s}$, annealing at $50{ }^{\circ} \mathrm{C}$ for $30 \mathrm{~s}$ and extension for $90 \mathrm{~s}$ at $70{ }^{\circ} \mathrm{C}$; followed by a final extension step at $70{ }^{\circ} \mathrm{C}$ for $10 \mathrm{~min}$. Following purification with sodium acetate $(\mathrm{pH} \mathrm{3.8;}$ $0.1 \mathrm{M}$ ) (Sambrook and Russell, 2001) the PCR products were sequenced in both directions using the SP-6 and T-7 primers, the BigDye ${ }^{\circledR}$ terminator v3.1 cycle sequencing kit (Applied Biosystems, Foster City, CA) and an ABI PRISM 377 automated DNA sequencer (Perkin Elmer, Warrington, United Kingdom).

\section{Structural organization and conserved regions in the MAT loci}

Sequence assembly, annotation and in silico translation were performed using CLC Bio Genomics Workbench (CLC Bio, Aarhus, Denmark). Sequences were aligned using ClustalW (Thompson et al., 1994), except for coding sequences, which were aligned using the codon-based algorithm of SQUINT (Goode and Rodrigo, 2007). The start of the flanking regions (i.e. the point at which homology is restored between MAT-1 and MAT-2 genomes) were identified manually by aligning the MAT1-1 and MAT1-2 sequences from the same species. Structural relationships were visualized with the graphical alignment program GATA (Nix and Eisen, 2005), based on the B12Seq algorithm (Tatusova and Madden, 1999), which enables identification of putative duplication and inversion events.

Evolutionarily conserved regions were identified using dnaSP version 5 (Librado and Rozas, 2009) by determining nucleotide diversity $(\pi)$ over a sliding window of $100 \mathrm{bp}$ and sliding in increments of $25 \mathrm{bp}$. Gene identification was performed using the online tools FGENESH (Salamov and Solovyev, 2000) and AUGUSTUS (Stanke et al., 2004). The 500 bp region upstream of each start codon was investigated to identify putative cis-regulatory transcription factor binding sites using 
MOTIFSEARCH (http://www.motif.genome.jp), based on the TRANSFAC database (Wingender $e t$ $a l .$, 1996), using the default cut-off value of $85 \%$ similarity. Only $500 \mathrm{bp}$ was used because this is roughly the size of most intergenic regions in the MAT locus.

\section{Phylogenetic relationships}

The two MAT idiomorphs were treated separately for phylogenetic analysis. All flanking DNA was excluded to avoid any dilution of phylogenetic signal due to recombination. Most appropriate nucleotide substitution models were chosen using jModelTest (Posada, 2008). Maximum likelihood phylogenetic trees were generated using PhyML 3.0 (Guindon et al., 2009) and internal branches were evaluated with 1000 bootstrap replicates. The $F$. oxysporum MAT idiomorph sequences (AB011379 and AB011378) were used as outgroups.

Competing tree topologies of $M A T$ and species trees were compared with respect to their fit to the sequence data using the Approximately Unbiased (AU) (Shimodaira, 2002) and Shimodaira and Hasegawa (1999) tests, as implemented in CONSEL (Shimodaira and Hasegawa, 2001). The two $M A T$ trees for the G. fujikuroi complex as well as the MAT tree for the F. graminearum complex generated by O'Donnell et al. (2004) were compared to their respective species trees, which were inferred by identifying consistently well-supported nodes in previous studies (O'Donnell et al., 1998, 2000, 2008; Kvas et al., 2009; Yli-Mattila et al., 2009). Only nodes supported by bootstrapvalues higher than $70 \%$ were retained for these analyses. Site likelihood values required by CONSEL were generated for each competing tree using Tree Puzzle (Schmidt et al., 2002).

\section{Sequence evolution in coding and non-coding regions}

To compare the nucleotide substitution rate in heterothallic and homothallic MAT loci, nucleotide diversity $(\pi)$ and its variance were measured using dnaSP. Fusarium sacchari was excluded from this analysis to avoid any skewing of the result by the highly divergent MAT1-1 sequence. Coding and non-coding portions of the locus were considered as separate datasets. To eliminate variation due to different evolutionary time-scales, each measurement of $\pi$ was divided by that generated for a sequence consisting of partial segments of house-keeping genes Translation Elongation Factor 1- $\alpha$ and Tubulin $\beta$-1 chain from the same set of species (accession numbers are listed in Table A2). This scaling procedure was based on the assumption that these house-keeping genes should experience comparable evolutionary rates in both heterothallic and homothallic species.

Using the available Fusarium genomes, variability in the MAT proteins was compared with an estimated genome-wide average. For this purpose, a set of amino acid sequences was generated using 100 randomly-selected protein-coding genes from the genomes of $F$. verticillioides (AAIM02000073), F. graminearum (NZ_AACM00000000), F. oxysporum (AAXH01000548) and $F$. circinatum. To avoid variation due to differences in gene annotation, alignments for genes with large gaps or non-homologous regions were not included in the analysis. The percentage identity values for the 100 proteins among the three species were compared with those for the five MAT proteins.

In order to consider the selective pressures affecting each $M A T$ gene, the likelihoods of various models of codon evolution were calculated using the CODEML program of the PAML package, version 4.3 (Yang, 2007). Each model imposed different constraints on $\omega$, which is the ratio of nonsynonymous substitutions per non-synonymous site to synonymous substitutions per synonymous site. We first investigated whether genes have experienced selective constraint significantly stronger than what could be expected under neutral evolution. A value of $\omega=1$ indicates an equal rate of synonymous and non-synonymous substitutions, implying neutrality. A neutral model in which $\omega=$ 1 was fixed, was compared to model M0 which allowed an estimated $\omega$ value. We then investigated 
whether positive selection drives $M A T$ gene evolution. Positive selection is expected to cause more non-synonymous than synonymous substitutions, resulting in $\omega>1$. However, since most proteins probably have some codons consistently under selective constraint, models that allowed codons to fall into various classes with different $x$ values were compared. Model M7 (the beta model) allowed a $\beta$ distribution of codons in the range $0 \leq \omega \leq 1$, while model M8 (the $\beta$ and $\omega$ model) allowed an additional class of $\omega \geq 1$. The likelihoods of these two pairs of models were compared using the likelihood ratio test (LRT), as described by Yang et al. (2000).

\section{Results}

\section{Structural organization and conserved regions in the $M A T$ loci}

Complete idiomorph sequences, including portions of both 50 and 30 flanks, were obtained for all 22 Fusarium isolates, except for the region at the 30 end of the F. sacchari MAT1-2 idiomorph. Because the rest of the derived idiomorph sequence for the same isolate was identical to that obtained by Arie et al. (1999), the missing sequence was also assumed to be identical. The length of the idiomorph (non-homologous region) ranged from $4.3 \mathrm{~kb}$ to $4.6 \mathrm{~kb}$ for MAT1-1 and $3.5 \mathrm{~kb}$ to 3.8 $\mathrm{kb}$ for $M A T 1-2$. GenBank accession numbers for $M A T$ sequences are pending.

In general, non-coding portions of the $M A T$ loci were found to be more variable between species than coding portions (Fig. 2). However, an area of low diversity was observed in the heterothallic MAT1-2 idiomorph, where no gene had previously been described (Fig. 2). Gene prediction using both FGENESH and AUGUSTUS revealed a predicted gene in this region with three exons. Using the predicted protein product for tBLASTn analysis (protein query against translated nucleotide database) against the complete genomes of $F$. verticillioides and $F$. oxysporum, both of which represent MAT-1 isolates, yielded no hits. In the homothallic F. graminearum genome however, there was a single significant hit to a predicted gene (FGSG 08894) found in an homologous location, immediately adjacent to MAT1-2-1 at the MAT locus, but in an inverted orientation. GATA analysis provided evidence of an inversion of this region in F. graminearum (Fig. 3). Gene expression data from $F$. graminearum (Güldener et al., 2006a), available via the $F$. graminearum database (FGDB) (Guldener et al., 2006b) verified that FGSG 08894 is transcribed. Furthermore, these results show a noticeable increase in transcript levels during the stages "in vitro sexual development" and "in vitro sexual development of Fusarium Cch1 calcium channel deletion mutant". This pattern was also observed in all of the four known MAT genes (Güldener et al., 2006a). The predicted MAT gene was designated MAT1-2-3. The name MAT1-2-2 has already been used to describe a different gene (Kanamori et al., 2007), which is not homologous to MAT1-2-3.

To gain insight into the range of species in which MAT1-2-3 may be present, a tBLASTn analysis against the GenBank database was performed. Apart from the Fusarium species mentioned herein, there were weak hits in two additional species in order Hypocreales; Cordyceps militaris (accession, AB084257) $(E=0.026)$ and Paecilomyces tenuipes (AB084921) $(E=0.31)$. Both hits corresponded to a position immediately adjacent to the described MAT1-2-1 gene, suggesting that these may be true orthologs.

All five MAT genes (MAT1-1-1, MAT1-1-2, MAT1-1-3, MAT1-2-1, and MAT1-2-3) had intact open reading frames (ORFs) of near-identical length among the eleven heterothallic Fusarium species, with two exceptions. In F. sacchari, MAT1-1-1 had a stop codon 11 codons upstream of that in the other species. This stop codon was present in both the sequence produced in this study as well as that published by Arie et al. (1999). This species probably encodes a slightly truncated MAT1-1-1 protein. In F. circinatum, the first exon of the newly-identified MAT1-2-3 gene was predicted to begin 126nt downstream of the start codon predicted in the other species, thus reducing the predicted protein by 42 amino acids. The upstream start codon is present in $F$. circinatum but a stop 
codon follows downstream. The presence of this stop codon was confirmed by PCR and Sanger sequencing. It is, therefore, unclear whether the $F$. circinatum prediction represents the true start codonfor all of these species or whether it has a different start codon to the others.
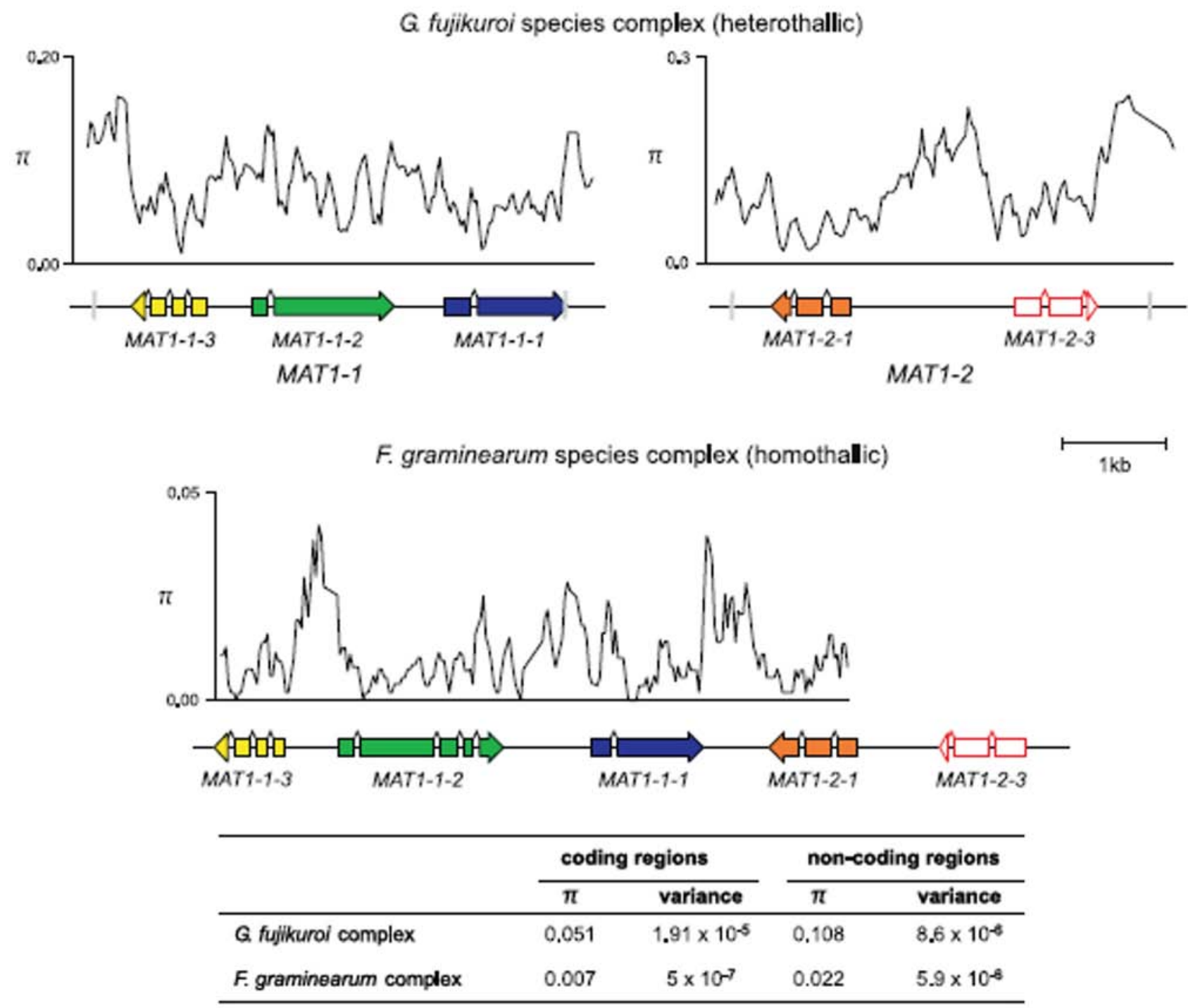

Figure 2. Structures and nucleotide diversity of Fusarium MAT loci. Boxes represent exons with the direction of transcription indicated. Gray lines separate idiomorphs from flanking regions, although these points varied among species. Plots indicate nucleotide diversity $(\pi)$, measured in a sliding window of $100 \mathrm{bp}$. For species in the G. fujikuroi complex, p was measured using sequences generated in this study. For the $F$. graminearum species complex, sequences generated by O'Donnell et al. (2004) were used, hence the plot only covers the region previously thought to constitute MAT locus.

In the upstream $500 \mathrm{bp}$ of the MAT genes, multiple motifs that appear to be homologous to the binding sites of eleven previously described transcription factors (most described in $S$. cerevisiae) were identified (Table A3). Multiple predicted binding sites of a heat shock factor (HSF), an alcohol dehydrogenase gene regulator (ADR1) and an activator of nitrogen-regulated genes (NIT2) were identified upstream of all genes, although the number of motifs sometimes differed among species. Several predicted bindings sites were identified upstream of a subset of the genes. For example, a stress response element (STRE) binding site was predicted upstream of all genes except MAT1-2-3. The most notable finding was the identification motifs similar to the binding sites of two yeast $M A T$ genes: MAT-Mc (Schizosaccharomyces pombe) and MATa2 (S. cerevisiae). In heterothallic species, all $M A T$ genes carried motifs similar to the MAT1-Mc binding site. In homothallic species only 
MAT1-1-1 carried this motif, the remaining four genes instead carried a motif similar to the MATa2 binding site (Table A3).



Figure 3. Homology between the F. graminearum and F. verticillioides MAT loci. The diagram was prepared from the output of GATA. Regions of strong homology are shaded and connected by lines. The intensity of shading indicates the strength of homology. Red shading with dashed lines indicates inverted homology. Genes are represented by box arrows. The $F$. graminearum $(F g)$ and $F$. verticillioides $(F v)$ MAT1-1 sequences were obtained from the genome sequences, while the $F$. verticillioides MAT1-2 sequence was obtained in this study.

\section{Phylogenetic relationships}

Phylogenetic analysis of both MAT1-1 and MAT1-2 sequences revealed three major clades for the G. fujikuroi isolates (Fig. 4). These correspond to the African, American and Asian clades described by O'Donnell et al. (1998). However the MAT1-1 idiomorph of F. sacchari did not fall into any of the three major clades, while the MAT1-2 idiomorph of this species grouped within the Asian clade as expected. The ML trees for MAT1-1 and MAT1-2 displayed several topological conflicts with the known species tree (Fig. 4). Comparison of competing topologies using the AU and SH tests revealed that the MAT loci did not support the known species trees over the topology of the ML tree (Table 2). Furthermore, neither MAT1-1 nor MAT1-2 supported the phylogeny representing the opposing idiomorph (Table 2). In contrast, for the homothallic F. graminearum complex, the MAT tree generated by O'Donnell et al. (2004) did not have a significantly better fit to the data than the species tree (Table 2).

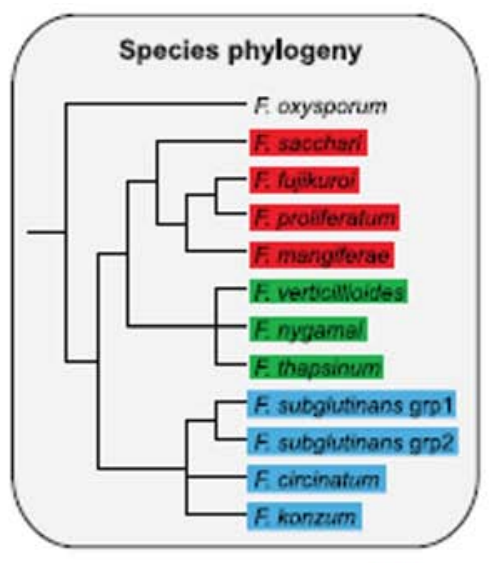

Asian clade
MAT1-1 ML tree (4380bp)

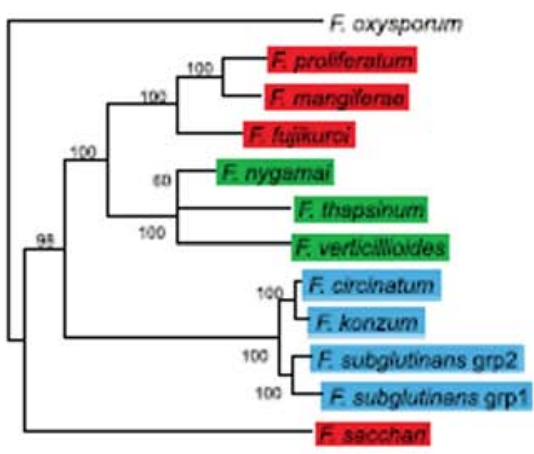

American clade

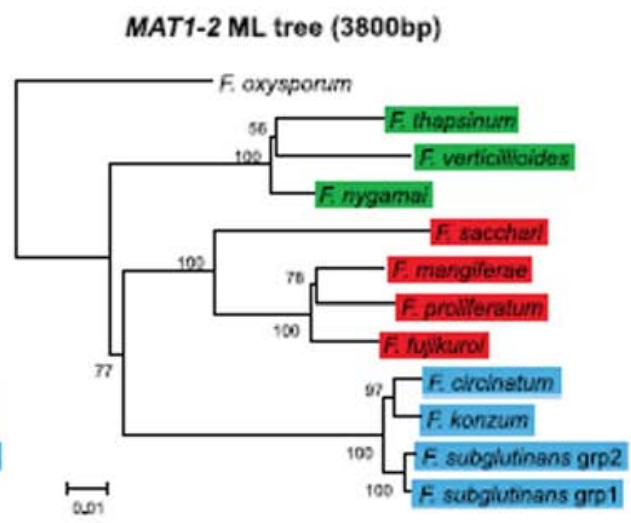

African clade

Figure 4. Maximum likelihood phylograms for G. fujikuroi complex MAT idiomorphs. The major clades of O'Donnell et al. (1998) are represented by colors. Numbers on branches indicate bootstrap values. The MAT1-1 and MAT1-2 phylogenies were inferred using, respectively, the general time 
reversible (GTR) model (Tavaré, 1986) and three-parameter (Kimura, 1981) model with unequal base frequencies (TPMluf; Posada 2008), both with gamma correction for among site rate variation.

Table 2. Results of the Approximately Unbiassed (AU) test of competing tree topologies.

\begin{tabular}{|c|c|c|c|c|}
\hline \multirow[t]{2}{*}{ Trees Tested $^{a}$} & \multicolumn{2}{|l|}{$P^{\mathrm{b}}$} & \multicolumn{2}{|c|}{ Rejected $^{c}$} \\
\hline & MAT-1 & MAT-2 & MAT-1 & MAT-2 \\
\hline \multicolumn{5}{|c|}{ G. fujikuroi complex species vs. MAT trees } \\
\hline Species & 0.000 & 0.034 & Yes & Yes \\
\hline MAT-1 ML tree & 1.000 & 0.000 & & Yes \\
\hline MAT-2 ML tree & 0.000 & 0.962 & Yes & \\
\hline \multicolumn{5}{|l|}{ Placement of $F$. sacchari } \\
\hline Within Asian clade & 0.009 & 1.000 & Yes & \\
\hline Outside Asian clade & 0.016 & 0.001 & Yes & Yes \\
\hline Outside G. fujikuroi complex & 0.993 & 0.000 & & Yes \\
\hline \multicolumn{5}{|c|}{ F. graminearum complex species vs. MAT trees } \\
\hline Species tree & 0.157 & & Yes & \\
\hline MAT tree & 0.843 & & & \\
\hline
\end{tabular}

a Alternative topologies being compared. Conflicts between trees are illustrated in Fig. 6.

b Approximately Unbiased (AU) test statistic. Results of the Shimodaira-Hasegawa $(\mathrm{SH})$ test are not shown as they echoed the AU results, but sometimes with lower significance.

${ }^{c}$ Likelihoods are significantly different at values of $P \leqslant 0.05$.

The unexpected grouping of the F. sacchari in the G. fujikuroi MAT1-1 tree was also addressed independently by comparing three trees that differed only in the position of $F$. sacchari. Placement of $F$. sacchari within the Asian clade could be rejected for the MAT1-1 idiomorph but not MAT1-2. Placement of $F$. sacchari outside of the Asian clade but within the G. fujikuroi complex could be rejected for both idiomorphs. However, placement of $F$. sacchari outside the complex could be rejected for MAT1-2 but not for MAT1-1. This implies that the most likely phylogenetic placement of the F. sacchari MAT1-1 idiomorph is outside of the G. fujikuroi complex.

\section{Sequence evolution in coding and non-coding regions}

Among the four species for which genome data was available (F. oxysporum, $F$. verticillioides, $F$. circinatum, and $F$. graminearum) all five $M A T$ genes displayed levels of percentage identity that were well below the average for 100 randomly-selected nuclear genes (Fig. 5). The MAT genes shared most similarity between the two $G$. fujikuroi complex species $F$. circinatum and $F$. verticillioides, and were most divergent when the three heterothallic species were compared with $F$. graminearum.

The results of the CODEML analyses indicated that all five genes in heterothallic species deviated significantly from the neutral model of evolution. By contrast, in homothallic species, MAT1-1-1 and MAT1-1-3, did not demonstrate a significant deviation from the neutral model. However, MAT1-1-1 displayed evidence for the presence of certain codons under positive selection in both heterothallic and homothallic species (Table A4). 


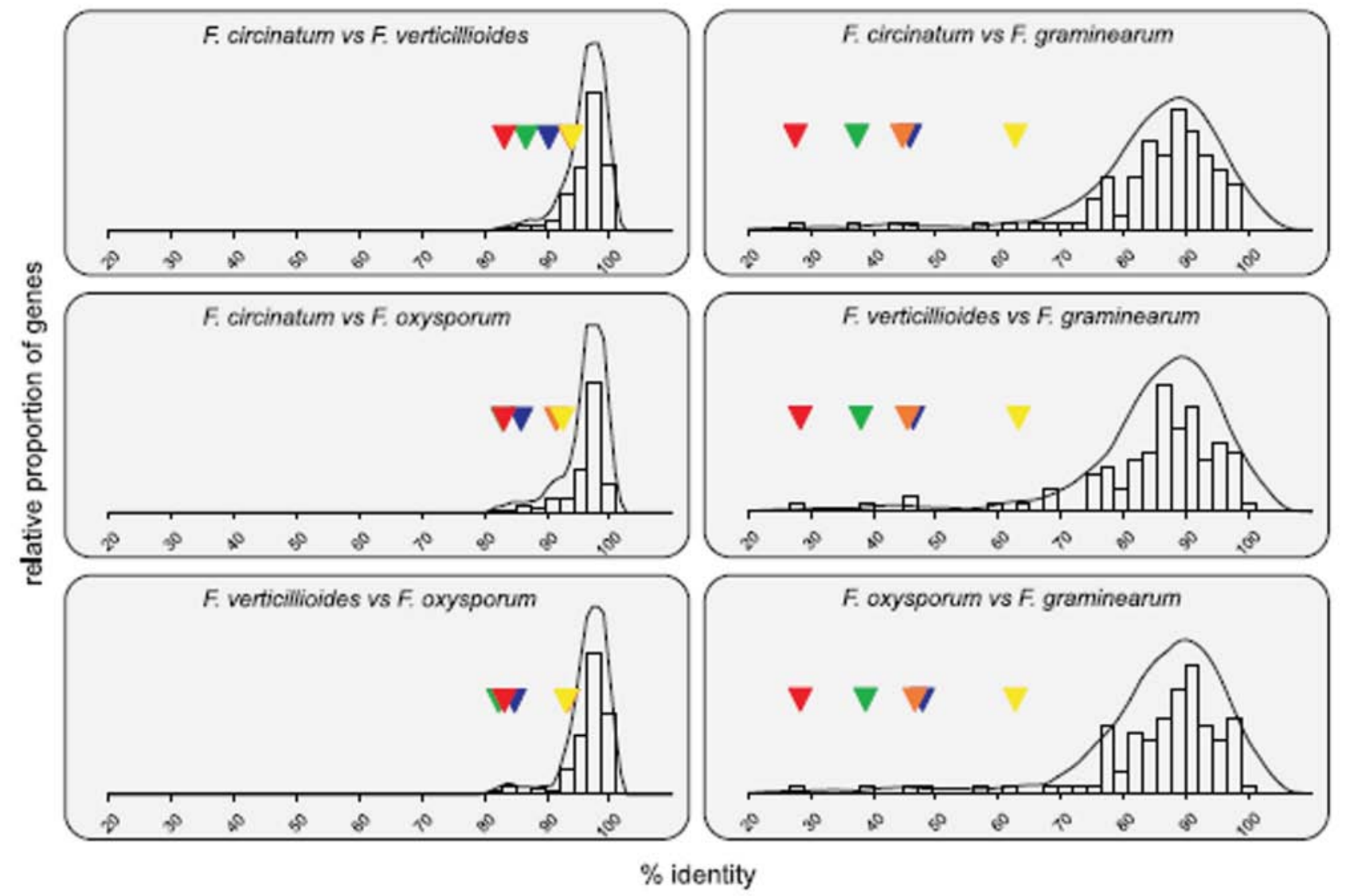

VMAT1-1-1 VMAT1-1-2 V MAT1-1-3 VMAT1-2-1 VMAT1-2-3

Figure 5. Scaled nucleotide diversity $(\pi)$ in coding and non-coding portions of MAT loci. Values of $\pi$ for from MAT loci were scaled based on the $\pi$ values of EF1- $\alpha$ and BT. Bars indicate the length of one standard deviation from the mean.

Scaled values of nucleotide diversity $(\pi)$ demonstrated that the coding regions of the MAT loci were comparatively less divergent among homothallic species (Fig. 6). However, the ratio of $\pi$ at nonsynonymous sites to $\pi$ at synonymous sites was similar in the heterothallic and homothallic species ( 0.253 and 0.243 , respectively). This indicated that the lower diversity in homothallic MAT genes was reflected at both non-synonymous and synonymous codon positions. The non-coding portion of the MAT locus was generally more divergent, but the relationship between the species complexes was reversed: non-coding portions were less divergent among heterothallic species (Fig. 6).

\section{Discussion}

To date, Fusarium spp. have been thought to carry the same complement of MAT genes as the model species Neurospora crassa (Arie et al., 1999; Yun et al., 2000; O'Donnell et al., 2004). This study has identified evidence of a previously unknown $M A T$ gene in what was previously thought to be a non-coding portion of the MAT1-2 idiomorph. The putative gene, tentatively named MAT1-2-3, appears to be homologous to FGSG 08894, which located adjacent to MAT1-2-1 in the $F$. graminearum genome. The predicted protein sequence of FGSG 08894 contains no known functional domains but the gene does display an expression profile in F. graminearum (Güldener et al., 2006a) that is similar to that of the other four $M A T$ genes, with significant up-regulation during the process of sexual development. Whether MAT1-2-3 is expressed in heterothallic Fusarium spp. remains to be verified. If so, the term "MAT gene" would be appropriate, because it is found only within the MAT1-2 idiomorph and is therefore absent from the genomes of MAT-1 individuals. The corresponding locations of MAT1-2-3 in heterothallic and homothallic species would be consistent 


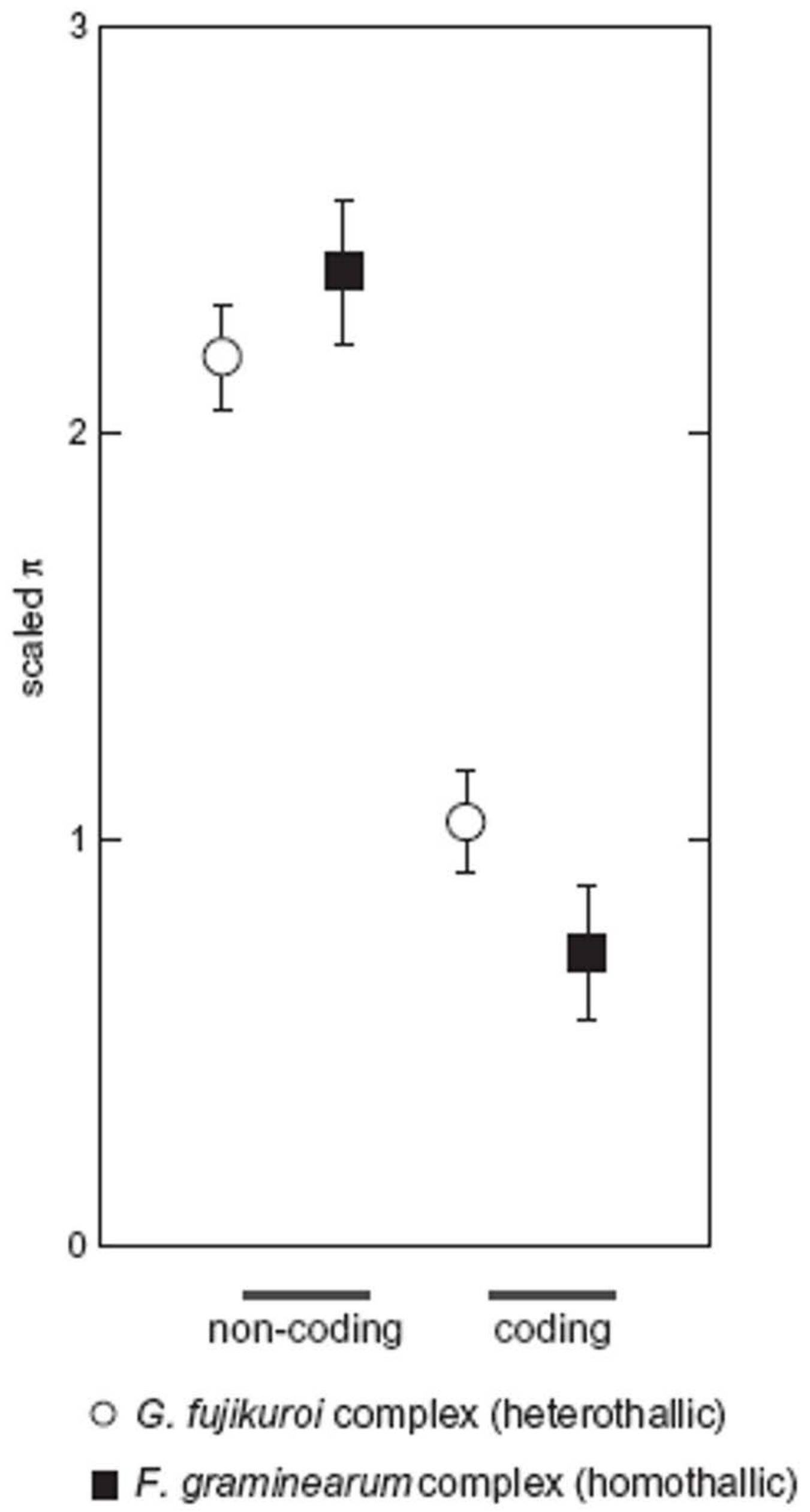


Figure 6. Amino acid percentage-identity of $M A T$ genes as compared to 100 nuclear genes from four genomes. Histograms indicate the distribution of percent identity for all 105 genes. Colored markers indicate the positions of the four MAT genes. The solid line indicates an estimated nonparametric kernel curve (Gasser et al. 1985) of density.

with the view of O'Donnell et al. (2004) that homothallism in F. graminearum arose through the fusion of heterothallic MAT loci. However, the gene's orientation is reversed, suggesting a segmental inversion of this region subsequent to the fusion. Putative homologs of MAT1-2-3 were also identified in two additional Hypocreales genomes. However, attempts to identify homologs using BLAST may be hindered by the rapid diversification of MAT genes as well as the fact that many Ascomycete genomes represent MAT-1 individuals.

A search for known binding sites of transcription factors found in model Ascomycetes revealed that a similar set of predicted binding sites appears consistently upstream of all five $M A T$ genes, including MAT1-2-3, which is further support for its authenticity. Conclusions with regard to the functions of the TFs involved should be made with caution; these TFs and their binding sites were described in species quite distantly related from Fusarium. Nevertheless, most TFs implicated here are involved (in their host organism) in processes consistent with a role in sexual reproduction. Most prevalent were predicted binding sites of TFs involved in response to environmental stresses such as the absence of certain nutrients or heat shock. Nutrient starvation is a well known stimulus of sexual development in Ascomycetes (Coppin et al., 1997; Pöggeler et al., 2006). Sexual development in Fusarium species is also known to be highly temperature-dependent (reviewed by Doohan et al., 2003). Whereas heat-shock per se has never been implicated in Fusarium; in Podospora anserina, a heat shock protein $\bmod -E$ is known to be necessary for sexual reproduction (Loubradou et al., 1997).

Probably the most surprising finding in the analysis of the regulatory regions was the identification of sites that appear to be homologous to the binding sites of certain yeast $M A T$ genes. In heterothallic species, all five MAT genes carried motifs similar to the binding site of MAT1-Mc. This is an HMG-domain protein found in the S. pombe MAT locus (Kjaerulff et al., 1997) that may be orthologous to MAT1-2-1 of filamentous species (Staben and Yanofsky, 1990). If this is the case, it is possible that these sites are in fact binding sites of the MAT1-2-1 HMG-domain protein, which would imply a form of self-regulation. In homothallic species, only one gene, MAT1-1-1, carried similar predicted $M A T 1-M c$ binding sites. The other four MAT genes instead carried motifs similar to the binding sites of MATa2. This is a homeodomain protein encoded by the $S$. cerevisiae MAT-a idiomorph (Johnson, 1995), of which there is no known ortholog in filamentous species. Whether these conserved motifs indeed correspond to actual TF binding sites will be an important focus for future research. Nevertheless, the fact that the two species complexes have many similarities but a few differences in the conserved motifs upstream of the various $M A T$ genes, implies that these regulatory regions have evolved, potentially reflecting adaptive changes in gene regulation.

Both MAT idiomorphs of species in the G. fujikuroi complex displayed significant support for phylogenies in conflict with the recognized species tree. It is possible that such incongruities could be the result of incomplete lineage sorting. However, this process requires the existence of polymorphism prior to speciation. Intraspecific polymorphisms in heterothallic MAT loci are thought to be rare and short-lived (Turgeon, 1998), possibly as a result of the lower effective population size. Another possible cause of the phylogenetic irregularities is inter-specific gene-flow. Similar findings in Neurospora led Strandberg et al. (2010) to propose that reproductive genes might be more prone to lateral gene-flow than others. This pattern could be driven by selection if the alleles that introgress from other species can restore sexuality to populations in which it has been lost. The potential to reproduce sexually can be lost through the rise of single-mating-type populations (in heterothallic species only) (e.g. Paoletti et al., 2006) or through genetic decay of 
reproductive genes (Strandberg et al., 2010; Wik et al., 2008). Indeed in Fusarium, many isolates display limited or no fertility (Kuhlman, 1982), the causes of which remain unknown. It is, therefore, possible that selection may have favored lateral transfer of functional MAT genes among Fusarium spp at certain points in their evolution.

The most intriguing phylogenetic aberration arising in this study was the grouping of the $F$. sacchari MAT1-1 idiomorph outside the G. fujikuroi complex, while the MAT1-2 idiomorph grouped in the Asian clade as expected. Further investigation using a larger dataset might improve phylogenetic resolution and rule out potential artifacts such as long-branch-attraction. The lateral transfer hypothesis appears doubtful because this would require a hybridization event between $F$. sacchari and a somewhat distant relative. Even within the G. fujikuroi complex, distinct mating populations (of which $F$. sacchari constitutes MP-B) are thought to be strongly reproductively isolated (Leslie, 1991; Leslie et al., 2004). Interestingly, this opinion did not prevail originally; early studies on $F$. sacchari suggested that it may indeed be cross-fertile with several other mating populations (Kuhlman, 1982). However, Britz et al. (1999) demonstrated that the progeny produced in such crosses, rather than being hybrids, were uniparental. This rather implies that $F$. sacchari is capable of homothallic reproduction. In light of the knowledge that $F$. sacchari has a heterothallic MAT locus and a MAT1-1 idiomorph that is highly dissimilar from others in the complex, these earlier claims should be re-visited.

Unlike the phylogenies of heterothallic MAT loci presented here and by Strandberg et al. (2010), the phylogeny of MAT loci in the homothallic F. graminearum complex generated by O'Donnell et al. (2004) contained few well-supported deviations from the species tree, and did not have a significantly better fit to the data. This could be taken as evidence that lateral gene-flow is less frequent in this homothallic lineage (despite apparently weak reproductive isolation O'Donnell et al. (2004)). However, in the homothallic MAT locus, introgressed alleles that do not become fixed might recombine with native alleles. This could make lateral transfer difficult to detect and might even cause some sequence homogenization among species. Indeed, O'Donnell et al. (2004) reported low inter-specific divergence and phylogenetic informativeness in MAT loci compared to other nuclear genes of the $F$. graminearum complex. In the current study, sequence divergence in coding portions of the MAT locus (relative to two house-keeping genes) was found to be lower in the homothallic than in the heterothallic complex. Nucleotide diversity was lower at both synonymous and non-synonymous sites, indicating that this was not the result of stronger purifying selection, which would only reduce diversity in the latter. These findings are therefore in agreement with the idea that homothallic $M A T$ genes that experience gene flow could diverge more slowly due to recombination between native and introgressed alleles.

In contrast with the coding sequences, non-coding portions of the MAT loci were more divergent among homothallic than heterothallic species. This finding also fits with the expectations given the differences in recombination. Heterothallic MAT loci do not recombine and all parts are therefore strongly linked. As a result, noncoding regions of heterothallic MAT loci are not independent, and could diverge more slowly due to functional constraint acting upon linked coding regions (Hudson, 1994). Free recombination in homothallic species would mean that the non-coding portions are more independent of any constraint affecting the genes.

Of the four available Fusarium genomes, only one was homothallic. Therefore, at the genome-wide level, a specific comparison between the rate of divergence among homothallic vs. heterothallic species was impossible. However, in general, MAT proteins were highly divergent compared to the estimate for the average protein. This is in agreement with the findings for Cochliobolus (Turgeon, 1998) and Neurospora (Wik et al., 2008) and also with the trend of rapid evolution in reproductive proteins (Civetta and Singh, 1998; Swanson and Vacquier, 2002; Clark et al., 2006). This rapid divergence of MAT genes over short evolutionary distances helps to explain their dramatic lack of 
conservation among genera and the difficulty experienced by researchers in identifying MAT loci in new species (Cisar et al., 1994; Arie et al., 1997).

Likelihood-based tests for selection affecting MAT genes, revealed evidence for the presence codons under positive diversifying selection in one gene, MAT1-1-1, in both species complexes. In homothallic species, MAT1-1-1 and MAT1-1-3 were also found to exhibit evolutionary patterns not significantly different from neutrality (lack of selective constraint). The reliability of this result is questionable, given the low frequency of inter-specific differences in the MAT genes in this complex. However, it does contradict O'Donnell et al. (2004) who reported the MAT genes to be under "strong purifying selection". The reason for this distinction is unclear but it might stem from the smaller dataset used here, which included only homothallic species from within the $F$. graminearum complex, with only a single sequence representing each species. Our results are, however, similar to those of Wik et al. (2008), who reported a lack of selective constraint affecting MAT genes of homothallic Neurospora spp. Unlike in Neurospora, there is no evidence that MAT genes are dispensable in homothallic Fusarium sp. (Lee et al., 2003). Furthermore, sexual reproduction cannot be considered a superfluous trait in homothallic Fusarium sp., in fact the sexual spores of $F$. graminearum provide an important means of dispersal and infection (Markell and Franch, 2003). However, homothallic species do retain the ability to outcross in a heterothallic manner, even in the absence of one functional MAT gene (Lee et al., 2003). This redundancy is a possible explanation for the observed relaxation of selective constraint in homothallic $M A T$ genes.

\section{Conclusion}

This study has provided new insights into the structure and evolution of the Fusarium MAT locus. Fusarium spp. appear to encode a novel MAT gene, MAT1-2-3, which may be absent from many other genera. Another noteworthy finding is the possibility that novel regulatory motifs could have been recruited by certain $M A T$ genes in the transition to homothallism, potentially allowing independent gene regulation. There is some phylogenetic evidence for lateral transfer of $M A T$ genes in Fusarium, and the MAT1-1 idiomorph of F. sacchari in particular will require further attention. There is little such evidence among homothallic species, although geneflow might be more difficult to detect in the face of recombination. In general Fusarium MAT proteins appear to evolve rapidly. One gene, MAT1-1-1, was found to be under positive diversifying selection. Again, recombination in homothallic $M A T$ loci could stem the rapid divergence if there is gene-flow. Some homothallic $M A T$ genes also display evidence for relaxed selective constraint, which may be associated with the partial functional redundancy. Overall these findings demonstrate that Fusarium MAT loci offer a useful model in which study many different aspects of molecular evolution.

\section{Acknowledgements}

We thank Dr. David Geiser for reading the manuscript and offering useful advice. This work was funded by The National Research Foundation (NRF), the DST (Department of Science and Technology)/NRF Centre of Excellence in Tree Health Biotechnology (CTHB), the University of Pretoria and the Tree Protection Cooperative Programme (TPCP).

\section{Appendix A. Supplementary material}

Supplementary material associated with this article can be found, in the online version, at doi:10.1016/j.fgb.2011.03.005. 


\section{References}

Arie, T., Christiansen, S.K., Yoder, O.C., Turgeon, B.G., 1997. Efficient cloning of ascomycete mating type genes by PCR amplification of the conserved MAT HMG Box. Fungal Genet. Biol. 21, $118-130$.

Arie, T., Yoshida, T., Shimizu, T., Kawabe, M., Yoneyama, K., Yamaguchi, I., 1999. Assessment of Gibberella fujikuroi mating type by PCR. Mycoscience 40, 311-314.

Birky, C.W., Walsh, J.B., 1988. Effects of linkage on rates of molecular evolution. Proc. Nat. Acad. Sci. USA 85, 6414-6418.

Britz, H., Coutinho, T.A., Wingfield, M.J., Marasas, W.F.O., Gordon, T.R., Leslie, J.F., 1999. Fusarium subglutinans f. sp. pini represents a distinct mating population in the Gibberella fujikuroi species complex. Appl. Environ. Microbiol. 65, 1198-1201.

Britz, H., Steenkamp, E.T., Coutinho, T.A., Wingfield, B.D., Marasas, W.F.O., Wingfield, M.J., 2002. Two new species of Fusarium section Liseola associated with mango malformation. Mycologia 94, 722-730.

Charlesworth, B., 2009. Fundamental concepts in genetics: effective population size and patterns of molecular evolution and variation. Nat. Rev. Genet. 10, 195-205.

Chaturvedi, V., Fan, J., Stein, B., Behr, M.J., Samsonoff, W.A., Wickes, B.L., Chaturvedi, S., 2002. Molecular genetic analyses of mating pheromones reveal intervariety mating or hybridization in Cryptococcus neoformans. Infect. Immun. 70, 5225-5235.

Cisar, C.R., TeBeest, D.O., Spiegel, F.W., 1994. Sequence similarity of mating type idiomorphs: a method which detects similarity among the Sordariaceae fails to detect similar sequences in other filamentous ascomycetes. Mycologia 86, 540-546.

Civetta, A., Singh, R.S., 1998. Sex-related genes, directional sexual selection, and speciation. Mol. Biol. Evol. 15, 901-909.

Clark, N.L., Aagaard, J.E., Swanson, W.J., 2006. Evolution of reproductive proteins from animals and plants. Reproduction 131, 11-22.

Coppin, E., Debuchy, R., Arnaise, S., Picard, M., 1997. Mating types and sexual development in filamentous ascomycetes. Microbiol. Mol. Biol. Rev. 61, 411-428.

Devier, B., Aguileta, G., Hood, M.E., Giraud, T., 2010. Using phylogenies of pheromone receptor genes in the Microbotryum violaceum species complex to investigate possible speciation by hybridization. Mycologia 102, 689-696.

Doohan, F.M., Brennan, J., Cooke, B.M., 2003. Influence of climatic factors on Fusarium species pathogenic to cereals. Eur. J. Plant Path. 109, 755-768.

Gasser, T., Muller, H.G., Mammitzsch, V., 1985. Kernels for nonparametric curve estimation. J. Roy Stat Soc. Ser. B (Meth.) 47, 238-252.

Glass, N.L., Grotelueschen, J., Metzenberg, R.L., 1990. Neurospora crassa a matingtype region. Proc. Nat. Acad. Sci. USA 87, 4912-4916. 
Glass, N.L., Smith, M.L., 1994. Structure and function of a mating-type gene from the homothallic species Neurospora africana. Mol. Gen. Genet. 244, 401-409.

Glass, N.L., Vollmer, S.J., Staben, C., Grotelueschen, J., Metzenberg, R.L., Yanofsky, C., 1988. DNAs of the two mating-type alleles of Neurospora crassa are highly dissimilar. Science 241,570 573.

Goode, M.G., Rodrigo, A.G., 2007. SQUINT: a multiple alignment program and editor. Bioinformatics 23, 1553-1555.

Güldener, U., Mannhaupt, G., Münsterkötter, M., Haase, D., Oesterheld, M., Stümpflen, V., Mewes, H.-W., Adam, G., 2006a. FGDB: A comprehensive fungal genome resource on the plant pathogen Fusarium graminearum. Nucleic Acids Res. 34, 456-458.

Güldener, U., Seong, K., Boddu, J., Cho, S., Trail, F., Xu, J., Adam, G., Mewes, H., Muehlbauer, G., Kistler, H., 2006b. Development of a Fusarium graminearum affymetrix GeneChip for profiling fungal gene expression in vitro and in planta. Fungal Genet. Biol. 43, 316-325.

Guindon, S., Delsuc, F., Dufayard, J.F., Gascuel, O., 2009. Estimating maximum likelihood phylogenies with PhyML. Met. Mol. Cell. Biol. 537, 113-137.

Hudson, R.R., 1994. How can the low levels of DNA sequence variation in regions of the Drosophila genome with low recombination rates be explained? Proc. Nat. Acad. Sci. USA 91, 6815-6818.

Johnson, A.D., 1995. Molecular mechanisms of cell-type determination in budding yeast. Curr. Opin. Genet. Dev. 5, 552-558.

Kanamori, M., Kato, H., Yasuda, N., Koizumi, S., Peever, T.L., Kamakura, T., Teraoka, T., Arie, T., 2007. Novel mating type-dependent transcripts at the mating type locus in Magnaporthe oryzae. Gene 403, 6-17.

Kimura, M., 1981. Estimation of evolutionary distances between homologous nucleotide sequences. Proc. Nat. Acad. Sci. USA 78, 454-458.

Kjaerulff, S., Dooijes, D., Clevers, H., Nielsen, O., 1997. Cell differentiation by interaction of two HMG-box proteins: Mat1-Mc activates M cell-specific genes in $S$. pombe by recruiting the ubiquitous transcription factor Ste11 to weak binding sites. EMBO J. 16, 4021-4033.

Klaasen, J.A., Nelson, P.E., 1996. Identification of a mating population, Gibberella nygamai sp. nov., within the Fusarium nygamai anamorph. Mycologia 88, 965-969.

Klittich, C.J.R., Leslie, J.F., 1992. Identification of a second mating population within the Fusarium moniliforme anamorph of Gibberella fujikuroi. Mycologia 84, 541-547.

Klittich, C.J.R., Leslie, J.F., Nelson, P.E., Marasas, W.F.O., 1997. Fusarium thapsinum (Gibberella thapsina): a new species in section Liseola from sorghum. Mycologia 89, 643-652.

Kronstad, J.W., Staben, C., 1997. Mating type in filamentous fungi. Ann. Rev. Genet. 31, 245-276.

Kuhlman, E.G., 1982. Varieties of Gibberella fujikuroi with Anamorphs in Fusarium Section. Mycologia 74, 759-768. 
Kvas, M., Marasas, W.F.O., Wingfield, B.D., Wingfield, M.J., Steenkamp, E.T., 2009. Diversity and evolution of Fusarium species in the Gibberella fujikuroi complex. Fungal Div. 34, 1-21.

Lee, J., Lee, T., Lee, Y.W., Yun, S.H., Turgeon, B.G., 2003. Shifting fungal reproductive mode by manipulation of mating type genes: obligatory heterothallism of Gibberella zeae. Mol. Microbiol. $50,145-152$.

Lee, S.C., Ni, M., Li, W., Shertz, C., Heitman, J., 2010. The evolution of sex: a perspective from the fungal kingdom. Microbiol. Mol. Biol. Rev. 74, 298-340.

Leslie, J., Zeller, K., Wohler, M., Summerell, B., 2004. Interfertility of two mating populations in the Gibberella fujikuroi species complex. Eur. J. Plant Path. 110, 611-618.

Leslie, J.F., 1991. Mating populations in Gibberella fujikuroi (Fusarium section Liseola). Phytopathology 81, 1058-1060.

Leslie, J.F., 1995. Gibberella fujikuroi: available populations and variable traits. Can. J. Bot. 73, $282-291$.

Leslie, J.F., Summerell, B.A., Bullock, S., Doe, F.J., 2005. Description of Gibberella sacchari and Neotypification of its Anamorph Fusarium sacchari. Mycologia 97, 718-724.

Librado, P., Rozas, J., 2009. DnaSP v5: A software for comprehensive analysis of DNA polymorphism data. Bioinformatics 25, 1451-1452.

Loubradou, G., Begueret, J., Turcq, B., 1997. A mutation in an HSP90 gene affects the sexual cycle and suppresses vegetative incompatibility in the fungus Podospora anserina. Genetics 147, 581588.

Markell, S.G., Francl, L.J., 2003. Fusarium head blight inoculum: species prevalence and Gibberella zeae spore type. Plant Dis 87, 814-820.

Nix, D., Eisen, M., 2005. GATA: a graphic alignment tool for comparative sequence analysis. BMC Bioinf. 6, 9.

O’Donnell, K., Cigelnik, E., Nirenberg, H.I., 1998. Molecular systematic and phylogeography of the Gibberella fujikuroi species complex. Mycologia 90, 465-493.

O’Donnell, K., Nirenberg, H.I., Aoki, T., Cigelnik, E., 2000. A multigene phylogeny of the Gibberella fujikuroi species complex: Detection of additional phylogenetically distinct species. Mycoscience 41, 61-78.

O’Donnell, K., Ward, T.J., Geiser, D.M., Corby Kistler, H., Aoki, T., 2004. Genealogical concordance between the mating type locus and seven other nuclear genes supports formal recognition of nine phylogenetically distinct species within the Fusarium graminearum clade. Fungal Genet. Biol. 41, 600-623.

O’Donnell, K., Ward, T.J., Aberra, D., Kistler, H.C., Aoki, T., Orwig, N., Kimura, M., Bjørnstad, A., Klemsdal, S.S., 2008. Multilocus genotyping and molecular phylogenetics resolve a novel head blight pathogen within the Fusarium graminearum species complex from Ethiopia. Fungal Genet. Biol. 45, 1514-1522. 
Paoletti, M., Buck, K.W., Brasier, C.M., 2006. Selective acquisition of novel mating type and vegetative incompatibility genes via interspecies gene transfer in the globally invading eukaryote Ophiostoma novo-ulmi. Mol. Ecol. 15, 249-262.

Pöggeler, S., Nowrousian, M., Kück, U., 2006. Fruiting-body development in ascomycetes. In: Kües, U., Fischer, R. (Eds.), Growth, Differentiation and Sexuality. Vol. 1 of The Mycota. Springer Berlin Heidelberg, Berlin/Heidelberg, (Chapter 16). pp. 325-355.

Posada, D., 2008. jModelTest: Phylogenetic model averaging. Mol. Biol. Evol. 25, 1253-1256.

Salamov, A.A., Solovyev, V.V., 2000. Ab initio gene finding in Drosophila genomic DNA. Genom. Res. 10, 516-522.

Sambrook, J., Russell, D., 2001. Molecular Cloning: A Laboratory Manual, third ed. Cold Spring Harbor Laboratory Press, Plainview, NY.

Schmidt, H.A., Strimmer, K., Vingron, M., von Haeseler, A., 2002. TREE-PUZZLE: maximum likelihood phylogenetic analysis using quartets and parallel computing. Bioinformatics 18, 502504.

Shimodaira, H., 2002. An approximately unbiased test of phylogenetic tree selection. Syst. Biol. 51, 492-508.

Shimodaira, H., Hasegawa, M., 1999. Multiple comparisons of log-likelihoods with applications to phylogenetic inference. Mol. Biol. Evol. 16, 1114.

Shimodaira, H., Hasegawa, M., 2001. CONSEL: for assessing the confidence of phylogenetic tree selection. Bioinformatics 17, 1246-1247.

Shiu, P.K.T., Glass, N.L., 2000. Cell and nuclear recognition mechanisms mediated by mating type in filamentous ascomycetes. Curr. Opin. Microbiol. 3, 183-188.

Staben, C., Yanofsky, C., 1990. Neurospora crassa a mating-type region. Proc. Nat. Acad. Sci. USA 87, 4917-4921.

Stanke, M., Steinkamp, R., Waack, S., Morgenstern, B., 2004. AUGUSTUS: A web server for gene finding in eukaryotes. Nucleic Acids Res. 32, W309-W312.

Steenkamp, E.T., Wingfield, B.D., Coutinho, T.A., Wingfield, M.J., Marasas, W.F.O., 1999. Differentiation of Fusarium subglutinans f. sp. pini by histone gene sequence data. Appl. Environ. Microbiol. 65, 3401-3406.

Steenkamp, E.T., Wingfield, B.D., Coutinho, T.A., Zeller, K.A., Wingfield, M.J., Marasas, W.F.O., Leslie, J.F., 2000. PCR-based identification of MAT-1 and MAT-2 in the Gibberella fujikuroi species complex. Appl. Environ. Microbiol. 66, 4378-4382.

Steenkamp, E.T., Wingfield, B.D., Desjardins, A.E., Marasas, W.F.O., Wingfield, M.J., 2002. Cryptic speciation in Fusarium subglutinans. Mycologia 94, 1032-1043.

Strandberg, R., Nygren, K., Menkis, A., James, T.Y., Wik, L., Stajich, J.E., Johannesson, H. Conflict between reproductive gene trees and species phylogeny among heterothallic and pseudohomothallic members of the filamentous ascomycete genus Neurospora. Fungal Genet. Biol. 47: 869-878. 
Swanson, W.J., Vacquier, V.D., 2002. The rapid evolution of reproductive proteins. Nat. Rev. Genet. $3,137-144$.

Tatusova, T.A., Madden, T.L., 1999. BLAST 2 Sequences, a new tool for comparing protein and nucleotide sequences. FEMS Microbiol. Lett. 174, 247-250.

Tavaré, S., 1986. Some Probabilistic and Statistical Problems in the Analysis of DNA Sequences. Amer Mathematical Society, vol. $17,57-86$.

Thompson, J.D., Higgins, D.G., Gibson, T.J., 1994. CLUSTAL w: Improving the sensitivity of progressive multiple sequence alignment through sequence weighting, position-specific gap penalties and weight matrix choice. Nucleic Acids Res. 22, 4673-4680.

Turgeon, B.G., 1998. Application of mating type gene technology to problems in fungal biology. Ann. Rev. Phytopath. 36, 115-137.

Turgeon, B.G., Yoder, O.C., 2000. Proposed nomenclature for mating type genes of filamentous ascomycetes. Fungal Genet. Biol. 31, 1-5.

Vicoso, B., Charlesworth, B., 2009. Recombination rates may affect the ratio of X to autosomal non-coding polymorphism in African populations of Drosophila melanogaster. Genetics 181, 16991701.

Wik, L., Karlsson, M., Johannesson, H., 2008. The evolutionary trajectory of the mating-type (mat) genes in Neurospora relates to reproductive behavior of taxa. BMC Evol. Biol. 8, 109.

Wingender, E., Dietze, P., Karas, H., Knüppel, R., 1996. TRANSFAC: A database on transcription factors and their DNA binding sites. Nucleic Acids Res. 24, 238-241.

Yang, Z., 2007. PAML 4: phylogenetic analysis by maximum likelihood. Mol. Biol. Evol. 24, 15861591.

Yang, Z.H., Nielsen, R., Goldman, N., Pedersen, A.M.K., 2000. Codon-substitution models for heterogeneous selection pressure at amino acid sites. Genetics 155, 431-449.

Yli-Mattila, T., Gagkaeva, T., Ward, T.J., Aoki, T., Kistler, H.C., O’Donnell, K., 2009. A novel Asian clade within the Fusarium graminearum species complex includes a newly discovered cereal head blight pathogen from the Russian Far East. Mycologia 101, 841-852.

Yun, S.H., Berbee, M.L., Yoder, O.C., Turgeon, B.G., 1999. Evolution of the fungal self-fertile reproductive life style from self-sterile ancestors. Proc. Nat. Acad. Sci. USA 96, 5592-5597.

Yun, S.-H., Arie, T., Kaneko, I., Yoder, O.C., Turgeon, B.G., 2000. Molecular organization of mating type loci in heterothallic, homothallic, and asexual Gibberella/Fusarium species. Fungal Genet. Biol. 31, 7-20.

Zeller, K.A., Summerell, B.A., Bullock, S., Leslie, J.F., 2003. Gibberella konza (Fusarium konzum) sp. nov. from prairie grasses, a new species in the Gibberella fujikuroi species complex.. Mycologia 95, 943-954. 